\title{
Quantitative Assessment of 12-Lead ECG Synthesis Using CAVIAR
}

\author{
Julie Ann Scherer, MSE, * Paul Rubel, PhD, $†$ Jocelyne Fayn, PhD, $\uparrow$ and \\ Jos L. Willems, MD, PhD‡
}

\begin{abstract}
The objective of this study is to assess the performance of patientspecific segment-specific (PSSS) synthesis in QRST complexes using CAVIAR, a new method of the serial comparison for electrocardiograms and vectorcardiograms. A collection of 250 multi-lead recordings from the Common Standards for Quantitative Electrocardiography (CSE) diagnostic pilot study is employed. QRS and ST-T segments are independently synthesized using the PSSS algorithm so that the mean-squared error between the original and estimated waveforms is minimized. CAVIAR compares the recorded and synthesized QRS and ST-T segments and calculates the mean-quadratic deviation as a measure of error. The results of this study indicate that estimated QRS complexes are good representatives of their recorded counterparts, and the integrity of the spatial information is maintained by the PSSS synthesis process. Analysis of the ST-T segments suggests that the deviations between recorded and synthesized waveforms are considcrably greater than those associatcd with the QRS complexes. The poorer performance of the ST-T segments is attributed to magnitude normalization of the spatial loops, low-voltage passages, and noise interference. Using the meanquadratic deviation and CAVIAR as methods of performance assessment, this study indicates that the PSSS-synthesis algorithm accurately maintains the signal information within the 12-lead electrocardiogram. Key words: 12-lead ECG, ECG synthesis, ECG serial analysis, CAVIAR.
\end{abstract}

For the last 25 years, several investigators have studied the synthesis of the 12-lead electrocardiogram (ECG) from a minimal lead set. For situations where patients experience transient cardiac symptoms, timely acquisition of a 12-lead ECG greatly benefits clinical evaluation. Often, recording one in

*From the Department of Electrical Engineering and Computer Science. The University of Michigan, Ann Arbor, Michigan.

†From the National Institute of Health and Medical Research, INSERM U121, Lyon, France.

$\neq$ From the Department of Medical Informatics, Katholieke Universiteit, Leuven, Belgium.

Supported by the Belgian-American Educational Foundation, INSERM, and the Flemish Regional Government.

Reprint requests: Julie Ann Scherer, Department of Electrical Engineering and Computer Science, 1301 Beal Avenue, The University of Michigan, Ann Arbor, MI 48109-2122. due time is difficult or impractical. Continuous acquisition of three pseudo-orthogonal signals and subsequent estimation of the 12-lead ECG can provide physicians with important information about the diagnostic changes in the electrical activity of the heart. Recent publications, which present a new synthesis method based on a patient-specific segment-specific (PSSS) algorithm, indicate accurate estimation of leads $\mathrm{V}_{1}, \mathrm{~V}_{3}, \mathrm{~V}_{4}, \mathrm{~V}_{5}$, and $\mathrm{V}_{6}{ }^{1-3}$ These evaluations use statistical measures, such as correlation coefficient and root mean squared error on a per lead basis, to determine the accuracy of the reproductions. ${ }^{1,3}$ Such statistics, however, fail to quantify completely errors that might affect the lead set as a whole. In particular, the synthesis process may cause time de- 
Table 1. The MQD Results Obtained From the QRS and ST-T Analysis

\begin{tabular}{|c|c|c|c|c|c|}
\hline \multirow[b]{2}{*}{ MQD $\mu \mathrm{V}$} & \multicolumn{2}{|c|}{ CSE $n=250$} & \multicolumn{2}{|c|}{ Healthy $n=720$} & \multirow{2}{*}{$\begin{array}{c}\text { Root-mean-squared } \\
\text { Noise } \mu V\end{array}$} \\
\hline & QRS & ST-T & QRS & ST-T & \\
\hline Mean & 34.6 & 44.0 & 56.0 & 42.1 & 9.3 \\
\hline Standard deviation & 22.5 & 44.9 & 30.6 & 19.9 & 4.8 \\
\hline 96th percentile & 84.8 & 143.4 & 120.8 & 79.0 & 23.8 \\
\hline Maximum & 142.5 & 356.3 & 190.2 & 257.3 & 29.1 \\
\hline
\end{tabular}

Mean-quadratic deviations between recorded and synthesized QRS complexes and ST-T segments for 250 patients.

lays or changes in the phase relations of the signals. A new method of serial comparison, called CAVIAR, measures the difference between a pair of optimally superimposed, spatial vector loops. ${ }^{4}$ A study has been undertaken to assess the performance of PSSS synthesis in QRST complexes using CAVIAR. For the QRS and ST-T segments, CAVIAR determines the mean-quadratic deviations between pairs of spatial loops and thus, provides another technique for quantitative assessment of ECG synthesis.

\section{Materials and Methods}

\section{Synthesis Methods}

Two methods of synthesis are applied to the 12lead ECGs. First, recorded PQRST complexes are estimated using the PSSS algorithm. ${ }^{1}$ These complexes were selected by the ECG Lyon analysis program as being representative of the mean. The PSSS technique divides the ECG into three waveforms, namely the PR segment, QRS complex, and the ST-T segment including the $\mathrm{T}$ wave. For each waveform, a set of estimators is determined that maps leads I, II, and $V_{2}$ to the remaining nine. These estimators are calculated from multiple linear regression models, which

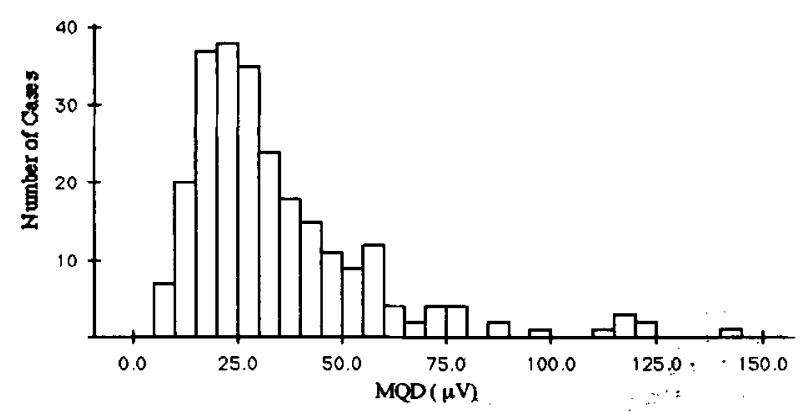

Fig. 1. Mean-squared deviations for the QRS synthesis in 250 patients. minimize the mean-squared error between the recorded and synthesized signals. Second, an orthogonal set of leads are predicted from the 12-lead ECG using the Levkov T3 transformation. ${ }^{5,6}$ This method predicts the Frank vectorcardiogram (VCG) using a single set of coefficients for all patients. These coefficients were calculated originally by Levkov using multivariate regression analysis on a group of 92 records. We chose the Levkov $\mathrm{T} 3$ transformation because previous investigations demonstrated that it maintains the spatial information of the VCG accurately and performs consistently regardless of the population. ${ }^{6}$ In our study, orthogonal representations are generated independently for the recorded and synthesized 12-lead sets to insure compatibility with the CAVIAR serial analysis program.

\section{Method of Performance Assessment}

CAVIAR is a method for directly comparing two three-dimensional VCG loops. ${ }^{4,6}$ The comparison is performed by optimally superimposing the spatio-

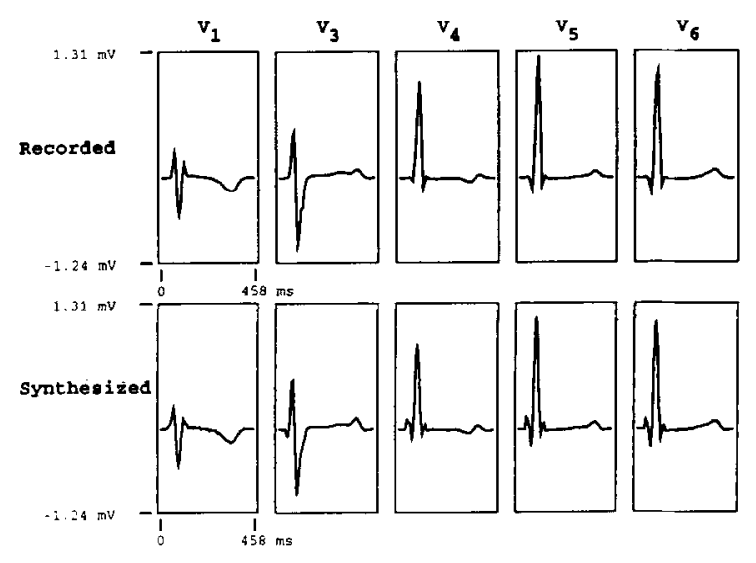

A

Fig. 2. (A) Recorded and synthesized QRST scgments for leads $\mathrm{V}_{1}, \mathrm{~V}_{3}, \mathrm{~V}_{4}, \mathrm{~V}_{5}$, and $\mathrm{V}_{6}$. (Figure continues) 


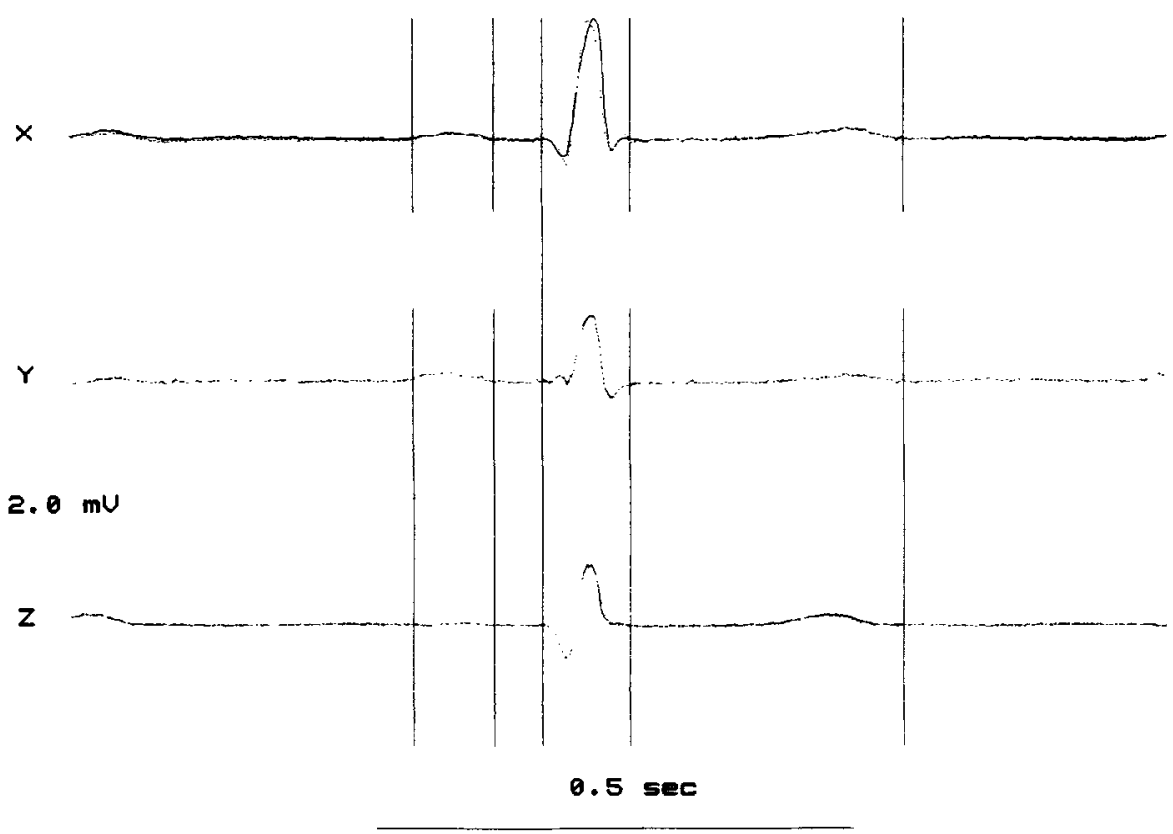

Fig. 2. (Continued) (B) XYZ signals derived from the 12 -lead ECG. $X$ signifies the synthesized $X Y Z$ from the recorded ECG. $S$ denotes the synthesized $X Y Z$ from the synthesized ECG. (C) CAVIAR analysis for the recorded and synthesized QRS complexes. X and S designate the recorded the synthesized ECGs, respectively.
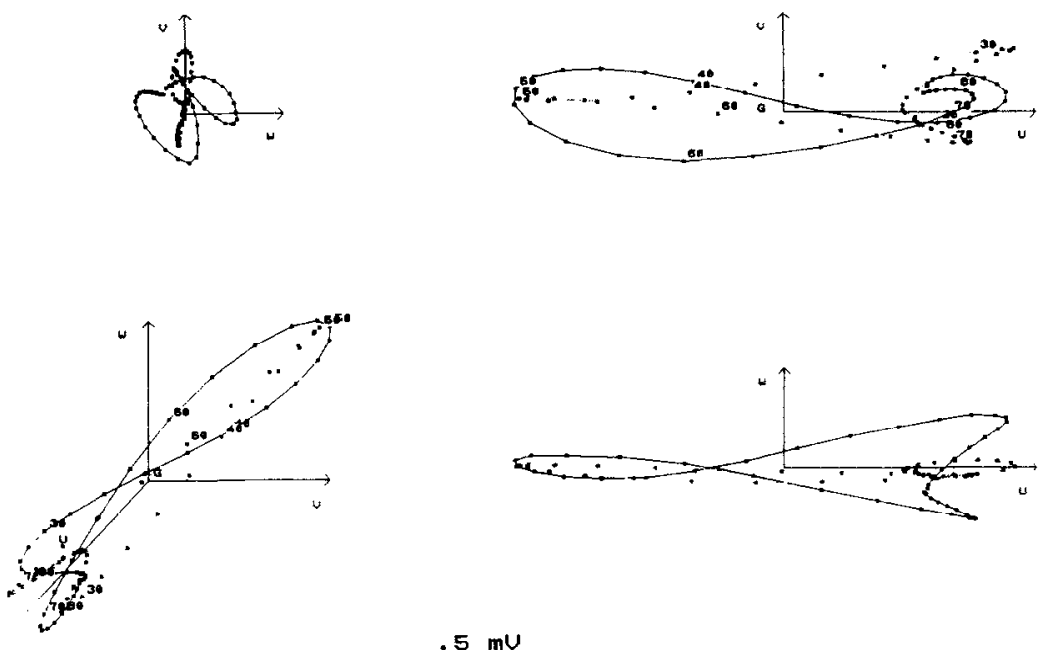

C

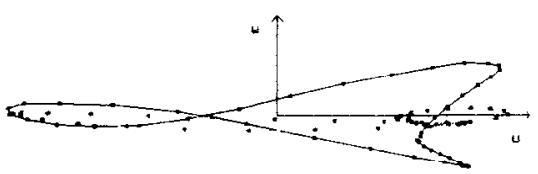

$5 \mathrm{mU}$

$\operatorname{MQD}(\mathrm{mmU})=118.90$

REF: -

temporal representations in an orthogonal coordinate system defined by the inertial axes of the loops. During this positioning process, resynchronizations and geometric transformations are performed to compensate for delineation errors and differences due to electrode displacements. The result is an optimal alignment for the two spatio-temporal images.
The mean-quadratic deviation (MQD), measured in microvolts, is used to quantify the differences between spatial loops. In this study, spatial loops are generated separately for the QRS complexes and ST-T segments of the recorded and synthesized ECGs. ${ }^{4,7} \mathrm{All}$ loops are normalized to a $1 \mathrm{mV}$ spatial magnitude. 


\section{Study Population}

The population employed for this study consists of 250 multi-lead recordings from the Common Standards for Quantitative Electrocardiography (CSE) diagnostic pilot study. ${ }^{8}$ The simultaneously recorded 15-lead data were acquired in two locations, Glasgow $(n=159)$ and Dublin $(n=91)$. The library of 250 recordings is comprised of 120 normals and 130 abnormals, which represent six classes of cardiac disorders. The ECGs were recorded on digital tape and digitized at $500 \mathrm{~Hz}$.

Differences between recorded and synthesized ECG loops are compared to the month-to-month variability for a healthy population reported by Rubel, et al. ${ }^{6}$ This population consists of 720 young men $(\mu, 19.9 \pm 1.6$ years) whose serial VCGs were recorded at rest within an interval of $6.0 \pm 3.3$ months. ${ }^{9}$

\section{Results}

Table 1 summarizes the MQD results obtained from the QRS and ST-T analyses for the CSE pilotstudy population. For comparative purposes, statistics for the root-mean-squared noise measurements in the CSE database and the month-to-month variability of the healthy population are also included.

The results shown in Table 1 indicate that estimated QRS complexes are good representatives of their recorded counterparts. This is evidenced by an accurate replication of the spatial description of the lead set in the majority of cases. Comparison of the QRS results with those for the 720 patient population of normals illustrates that the mean value of $34.6 \mu \mathrm{V}$ and standard deviation of $22.5 \mu \mathrm{V}$ for our study falls below the month-to-month variability of the healthy population $(\mu, 56.0 \mu \mathrm{V} ; \sigma, 30.6 \mu \mathrm{V})$. Further analysis demonstrates that the maximum MQD of $142.5 \mu \mathrm{V}$ for our study compares favorably with the similar statistic reported for the 720 patient population (maximum, $190.2 \mu \mathrm{V}$ ). Displayed in the last column of Table 1 are the statistics for the root-mean-squared noise measurements. Noise is measured as the rootmean-squared amplitude in a $20 \mathrm{~ms}$ interval immediately preceding the QRS onset.

Figure 1 illustrates a histogram of the MQD values for the QRS reproductions in the 250 patients. As indicated by the statistics in Table 1 , the majority of cases fall below $35 \mu \mathrm{V}$. Eight cases have MQDs above $95 \mu \mathrm{V}$. Of these outlying cases, seven have diagnosed inferior, anterior, or combined myocardial infarction, or left ventricular or biventricular hypertrophy. Only one is normal based on cardiac catheterization.

Of the 250 cases, only two demonstrate changes in the phase relationships of the precordial leads due to the QRS estimation process. Figure 2 illustrates the recorded and reconstructed ECGs, derived VCGs, and CAVIAR analysis for one of these cases. In the QRS complexes of Figure 2A, the most noticeable differences between the recorded and synthesized beats are the additional deflections before the $Q$ waves in leads $V_{4}, V_{5}$, and $V_{6}$ and the enlarged $Q$ waves in leads $V_{3}, V_{4}, V_{5}$, and $V_{6}$. These artifacts are caused by an inaccurate estimation of the beginning of the QRS complex. In the ECG signals, however, the time advance of the estimated $R$ wave is not noticeable. The XYZ leads derived from the recorded and synthesized ECGs using the Levkov $\mathrm{T} 3$ transformation are shown in Figure 2B. The change in timing of the $\mathrm{R}$ wave is evident in lead $\mathrm{X}$. Figure $2 \mathrm{C}$ presents the CAVIAR output from the comparison of the recorded and synthesized QRS loops. The phase shift in the signal set produces significant changes in the spatial loop. The MQD of $118 \mu \mathrm{V}$ indicates that the advance of the $R$ wave and the synthesis artifacts prior to it contribute to an alteration of the spatial information of the 12-lead ECG.

Analysis of ST-T segments indicates that MQD values are higher than those associated with QRS complexes. Comparisons of the ST-T results for the CSE and healthy populations in Table 1 show that the mean MQD is slightly higher for the synthesized ECGs $(44.0 \mu \mathrm{V}$ vs $42.3 \mu \mathrm{V})$. The standard deviation of $44.9 \mu \mathrm{V}$ and the maximum MQD of $356.3 \mu \mathrm{V}$ for the CSE population suggest that the differences between the recorded and synthesized signal sets are

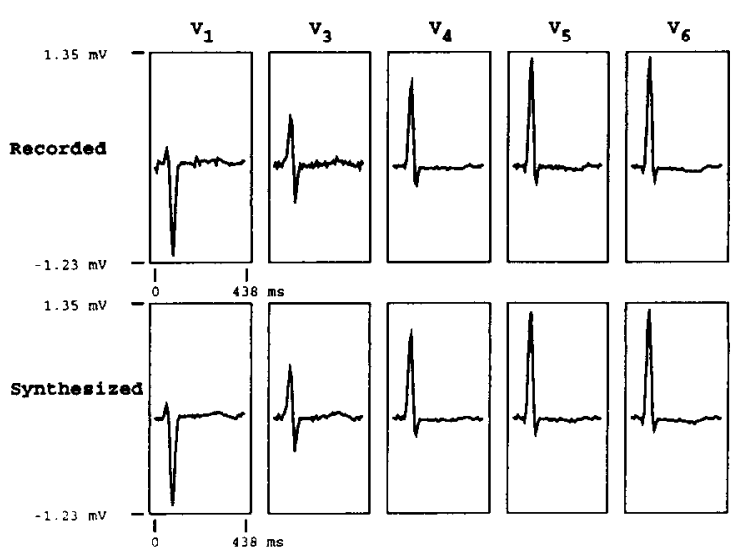

A

Fig. 3. (A) Recorded and synthesized QRST segments for leads $\mathrm{V}_{1}, \mathrm{~V}_{3}, \mathrm{~V}_{4}, \mathrm{~V}_{5}$, and $\mathrm{V}_{6}$. (Figure continues) 
Fig. 3. (Continued) (B) XYZ signals derived from the 12-lead ECG. $X$ signifies the synthesized XYZ from the recorded ECG. $S$ denotes the synthesized XYZ from the synthesized ECG. (C) CAVIAR analysis for the recorded and synthesized ST-T segments. $X$ and S designate the recorded and synthesized ECGs, respectively.

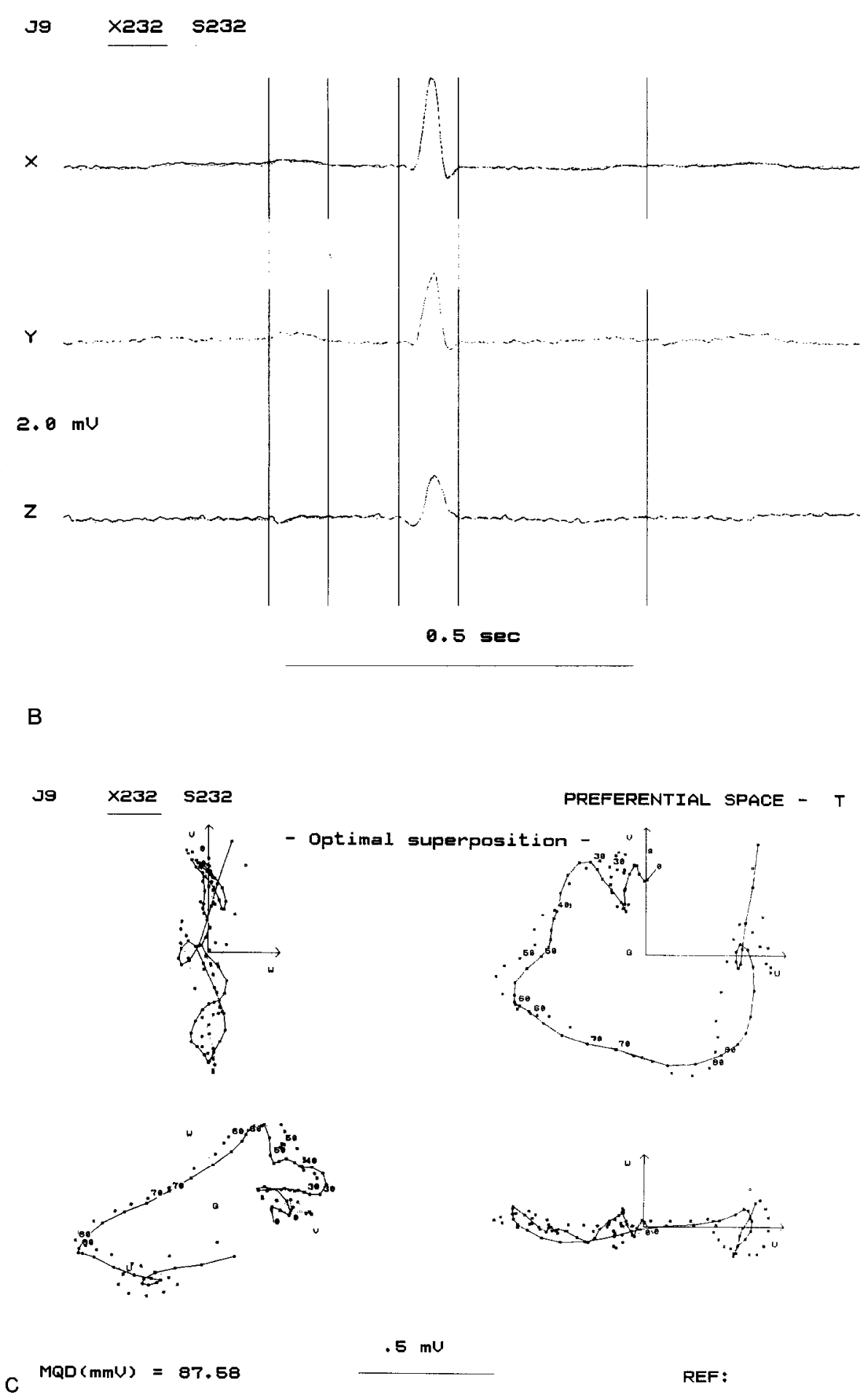

greater than the month-to-month limits in variability for the healthy population $(\sigma, 20.0 \mu \mathrm{V}$; maximum, $257.0 \mu \mathrm{V}$ ). The poorer performance of ST-T segment estimation is attributed to amplitude normalization of the spatial loops, low-voltage passages, and high root-mean-squared noise levels as shown in the last column of Table 1 .

Figure 3 displays the ECG signals, derived VCGs, and CAVIAR analysis for the ST-T segment of an exemplary case. Two major difficulties in estimating this waveform are apparent from this figure. Noise interference of $7.5 \mu \mathrm{V}$ masks the underlying ST-T segments and greatly impedes the estimation process. This problem is compounded by low voltages in all of the ST-T waveforms. Despite these difficulties, the estimated ST-T segments appear similar to their 
recorded counterparts. The XYZ leads in Figure 3B also illustrate the similarity between the recorded and synthesized waveforms, as well as the noise interference and low-amplitude passages. The initial $40 \mathrm{~ms}$ of the spatial loop in Figure $3 \mathrm{C}$ further exemplify the interference problem. The MQD of 87.58 $\mu \mathrm{V}$ suggests greater deviation than is recognizable in the scalar leads. This is because small deviations in low-amplitude waveforms are exaggerated by the 1 $\mathrm{mV}$ normalization of the spatial loops.

\section{Conclusions}

This study confirms that the PSSS-synthesis process does not effect time shifts or changes in the phase relationships between ECG signals in a majority of cases. When the results from this study are juxtaposed with the month-to-month variability of a normal population, the differences between recorded and synthesized QRS complexes are less than changes occurring over time in a healthy population. For the ST-T segments, the differences are greater than for their QRS counterparts. The MQD values for the ST-T segments may be misleading since they are measured from the normalized spatial loops. Hence, small alterations in low-amplitude ST-T segments will be associated with greater MQDs than similar variations in the QRS complexes.

The objective of 12-lead ECG synthesis is to provide the breadth of information available in the 12lead ECG while acquiring data from a smaller lead set. Such a technique may be useful for patient monitoring, particularly in coronary intensive care units, ambulatory ECGs, and telemetry systems. Results from this comparative study indicate that 12-lead ECG synthesis is accurate in maintaining spatial cardiac information contained in the QRS complexes of the lead set.

\section{References}

1. Scherer JA, Nicklas JM: Synthesis of the 12-lead electrocardiogram from a 3 lead semiorthogonal subset using patient-specific transformation arrays. p. 449. In Ripley KL (ed): Computers in cardiology, IEEE Computer Society, Washington, DC, 1988

2. Scherer JA, Monsur J, Nicklas JM: 12-lead ECG synthesis in 100 patients using patient-specific/segment-specific transformation vectors. J Am Coll Cardiol 15: 191A, 1990

3. Scherer JA, Jenkins JM, Nicklas JM: Synthesis of the 12-lead electrocardiogram from a 3-lead subset using patient-specific transformation vectors: an algorithm approach to computerized signal synthesis. J Electrocardiol 22(suppl):S128, 1989

4. Fayn J, Rubel P: CAVIAR: a serial ECG processing system for the comparative analysis of VCGs and their interpretation with auto-reference to the patient. J Electrocardiol 21 (suppl):SI73, 1988

5. Levkov Ch L: Orthogonal electrocardiogram derived from the limb and chest electrodes of the conventional 12-lead system. Med Biol Eng Comput 25:155, 1987

6. Rubel P, Benhadid I, Fayn J: Quantitative assessment of 8 different for synthesizing Frank VCGs from simultaneously recorded standard ECG leads. J Electrocardiol 24(suppl):S I97, 1991

7. Rubel P, Fayn J, Mohsen N, Girard P: New methods of quantitative assessment of the extent and significance of serial ECG changes of the repolarization phase. $J$ Electrocardiol 21(suppl):S177, 1988

8. Willems JL, Abreu-Lima C, Arnaud P et al: Testing the performance of ECG computer programs: the CSE diagnostic pilot study. J Electrocardiol 20(suppl):S306, 1987

9. Rubel P, Fayn J, Forlini MC, Arnaud P: Stability of serial VCG parameters among a normal male population recorded before and after physical training. p. 239. In Ripley KL (ed): Computers in cardiology. IEEE Computer Society, Long Beach, 1987 